\title{
Using Millimeter Vlbi to Constrain Riaf Models of Sagittarius A*
}

\section{Citation}

Fish, Vincent L., Avery E. Broderick, Sheperd S. Doeleman, and Abraham Loeb. 2009. "USING MILLIMETER VLBI TO CONSTRAIN RIAF MODELS OF SAGITTARIUS A*." The Astrophysical Journal 692 (1): L14-18. https://doi.org/10.1088/0004-637x/692/1//14.

\section{Permanent link}

http://nrs.harvard.edu/urn-3:HUL.InstRepos:41393145

\section{Terms of Use}

This article was downloaded from Harvard University's DASH repository, and is made available under the terms and conditions applicable to Other Posted Material, as set forth at http:// nrs.harvard.edu/urn-3:HUL.InstRepos:dash.current.terms-of-use\#LAA

\section{Share Your Story}

The Harvard community has made this article openly available.

Please share how this access benefits you. Submit a story.

Accessibility 


\title{
USING MILLIMETER VLBI TO CONSTRAIN RIAF MODELS OF SAGITTARIUS A*
}

\author{
Vincent L. Fish ${ }^{1}$, Avery E. Broderick ${ }^{2}$, Sheperd S. Doeleman $^{1}$, and Abraham Loeb ${ }^{3}$ \\ ${ }^{1}$ Massachusetts Institute of Technology, Haystack Observatory, Route 40, Westford, MA 01886, USA; vfish@ haystack.mit.edu, dole@haystack.mit.edu \\ ${ }^{2}$ Canadian Institute for Theoretical Astrophysics, University of Toronto, 60 St. George St., Toronto, ON, M5S 3H8 Canada; aeb@cita.utoronto.ca \\ ${ }^{3}$ Institute for Theory and Computation, Harvard University, Center for Astrophysics, 60 Garden St., Cambridge, MA 02138, USA; aloeb@cfa.harvard.edu \\ Received 2008 September 29; accepted 2008 December 17; published 2009 January 21
}

\begin{abstract}
The recent detection of Sagittarius $\mathrm{A}^{*}$ at $\lambda=1.3 \mathrm{~mm}$ on a baseline from Hawaii to Arizona demonstrates that millimeter wavelength very long baseline interferometry (VLBI) can now spatially resolve emission from the innermost accretion flow of the Galactic center region. Here, we investigate the ability of future millimeter VLBI arrays to constrain the spin and inclination of the putative black hole and the orientation of the accretion disk major axis within the context of radiatively inefficient accretion flow (RIAF) models. We examine the range of baseline visibility and closure amplitudes predicted by RIAF models to identify critical telescopes for determining the spin, inclination, and disk orientation of the Sgr A* black hole and accretion disk system. We find that baseline lengths near $3 \mathrm{G} \lambda$ have the greatest power to distinguish amongst RIAF model parameters, and that it will be important to include new telescopes that will form north-south baselines with a range of lengths. If an RIAF model describes the emission from Sgr A*, it is likely that the orientation of the accretion disk can be determined with the addition of a Chilean telescope to the array. Some likely disk orientations predict detectable fluxes on baselines between the continental United States and even a single 10-12 $\mathrm{m}$ dish in Chile. The extra information provided from closure amplitudes by a four-antenna array enhances the ability of VLBI to discriminate amongst model parameters.
\end{abstract}

Key words: accretion, accretion disks - black hole physics - Galaxy: center - submillimeter - techniques: interferometric

\section{INTRODUCTION}

The Galactic center radio source Sagittarius A* is believed to be associated with a black hole with a mass of approximately $4 \times 10^{6} M_{\odot}$ at a distance of about $8 \mathrm{kpc}$ (Schödel et al. 2003; Ghez et al. 2008). The event horizon of Sgr A* has the largest apparent angular size of all known black holes, as viewed from the Earth. High angular resolution is critical to understanding $\mathrm{Sgr} \mathrm{A}^{*}$ because the size scales are so small: for instance, the apparent lensed horizon size is $55 \mu$ as. The quest for angular resolution has driven observers toward very long baseline interferometry (VLBI) at millimeter wavelengths. Most recently, Doeleman et al. (2008b) detected Sgr A* at $230 \mathrm{GHz}(\lambda=1.3 \mathrm{~mm})$ on baselines between the James Clerk Maxwell Telescope (JCMT) in Hawaii and the Submillimeter Telescope Observatory (SMTO) in Arizona, as well as between the SMTO and a Combined Array for Research in Millimeterwave Astronomy (CARMA) telescope in California. The former is the longest baseline ( $3.5 \mathrm{G} \lambda$, fringe spacing $\sim 60 \mu$ as) on which $\mathrm{Sgr} \mathrm{A}^{*}$ has ever been detected.

In the absence of a true ab initio simulation of Sgr A*'s accretion flow, a variety of physically simplified models have been proposed. Constraints from the observed spectrum, polarization, and variability have not proved sufficient to conclusively establish the nature of the emission region. This is evidenced by the continuing vigorous debate over the morphology of this region, e.g., primarily from the innermost regions of an inefficient accretion flow (Yuan et al. 2003), the acceleration region of a nascent jet (Falcke et al. 2000), or something qualitatively different (Igumenshchev et al. 2003). However, millimeter VLBI's ability to resolve the emitting region directly promises to discriminate amongst these. To do so will require a detailed understanding of the role that key physical parameters (such as black hole spin, disk/jet orientation, etc) play in shaping the millimeter images of Sgr A*. Conversely, if the context of the emission can be conclusively established, millimeter VLBI has great potential to extract these parameters.

In a related work, Broderick et al. (2008) examine the ability of the Doeleman et al. (2008b) VLBI detections to discriminate amongst various radiatively inefficient accretion flow (RIAF) models parameterized by black hole spin, viewing angle, and disk orientation. A key finding is that the detected flux on the JCMT-SMTO baseline argues against orientations in which the assumed accretion disk is close to face on. However, the RIAF parameters are strongly coupled, and there are not yet enough millimeter VLBI measurements to make strong statements about the black hole spin, for instance. In this work, we explore the question of which future millimeter VLBI observations are likely to have the greatest impact for distinguishing between RIAF models and are therefore most likely to determine the physical parameters of the black-hole/accretion-disk system.

\section{DISK MODELS AND TELESCOPES}

Here we consider an ensemble of RIAF models similar to that described in Yuan et al. (2003) and described in detail in Doeleman et al. (2008a) and Broderick et al. (2008). These are generally characterized by the inefficient energy transfer between the electrons and the ions. In particular, we model the millimeter flux from Sgr A* as synchrotron emission due to populations of thermal (though with considerably lower temperatures than the ions) and nonthermal electrons in a geometrically thick, quiescent disk containing a toroidal magnetic field. The thermal and nonthermal electron densities and thermal electron temperature are solved for using measured radio, submillimeter, and near-infrared fluxes as constraints. Model images as viewed from the Earth are produced using fully general relativistic radiative transfer.

Each model has a total flux density of $2.4 \mathrm{Jy}$ at $\lambda=1.3 \mathrm{~mm}$, in line with the integrated flux density measured by Doeleman 




Figure 1. Map of standard deviation of predicted RIAF model visibilities $(\sigma(u, v))$ at $\lambda=1.3 \mathrm{~mm}$ holding $\xi=0^{\circ}$ constant. Units are in $\mathrm{Jy}$. The largest spread in predicted model quantities occurs when the projected baseline is aligned with the disk major axis. Rotating the image on the plane of the sky produces a rotation of the visibility amplitudes in the $(u, v)$ plane.

et al. (2008b). These models are parameterized by black hole $\operatorname{spin}(a)$, inclination $\left(\theta=0^{\circ}\right.$ for a face-on disk), and orientation of the disk major axis on the plane of the sky $(\xi)$ measured counterclockwise from east (equivalently the position angle of the spin axis). (Henceforth we shall use the word "models" to refer to RIAF models with these three free parameters.) Models are produced at spins from $a=0.0$ to 0.9 in increments of 0.1 , as well as at 0.99 and 0.998 . The ensemble includes models at $\theta=1^{\circ}$ and then at $\theta=10^{\circ}$ to $90^{\circ}$ in $10^{\circ}$ increments. This particular ensemble is described in greater detail in Broderick et al. (2008). We consider models with $0^{\circ} \leqslant \xi<180^{\circ}$ with an increment of $\Delta \xi=5^{\circ}$ (in Section 3.1) or $30^{\circ}$ (in Sections 3.2 and 3.3). Visibility amplitudes are symmetric under $180^{\circ}$ rotation for any real intensity distribution.

We consider seven potential stations for millimeter VLBI: Hawaii, consisting of one or more of the JCMT, Submillimeter Array (SMA), and Caltech Submillimeter Observatory (CSO) possibly phased together into a single aperture; the SMTO on Mount Graham, Arizona; CARMA telescopes in California, either individually or phased together; the Large Millimeter Telescope (LMT) on Sierra Negra, Mexico; a Chilean station consisting of either the Atacama Submillimeter Telescope Experiment (ASTE), Atacama Pathfinder Array (APEX), or a phased array of Atacama Large Millimeter Array (ALMA) dishes; the Institut de radioastronomie millimétrique (IRAM) $30 \mathrm{~m}$ dish on Pico Veleta (PV), Spain; and the IRAM Plateau de Bure (PdB) Interferometer, phased together as a single aperture. Assumed telescope capabilities and sensitivities are detailed in Doeleman et al. (2008a).

\section{BLACK HOLE PARAMETER ESTIMATION}

\subsection{Favored Regions of the $(u, v)$ Plane}

For the purpose of distinguishing different models, it is most desirable to obtain observations of Sgr A* on baselines at points at which the visibilities are most disparate. We compute model visibilities

$$
V(u, v)=\iint d x d y I(x, y) e^{-2 \pi i(x u+y v) / \lambda},
$$

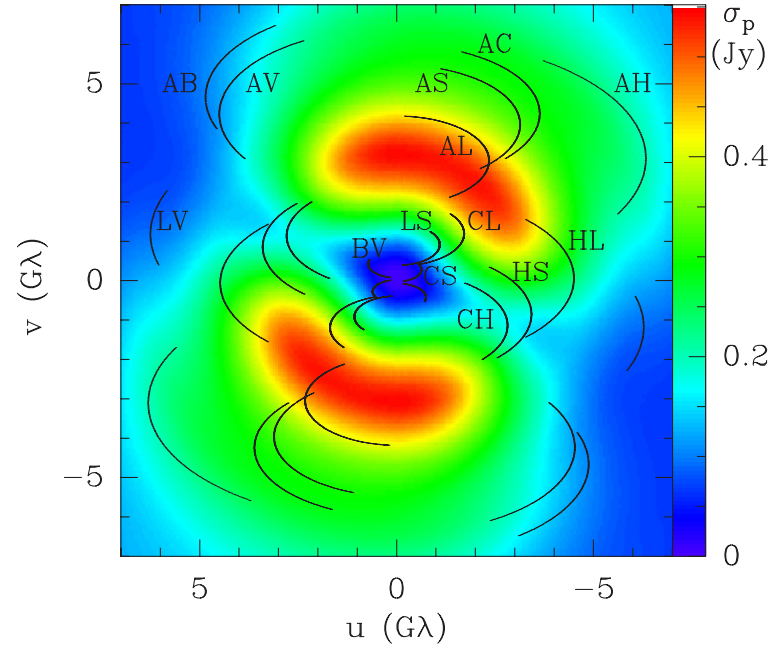

Figure 2. Plot of the standard deviation $\sigma_{p}(u, v)$ restricted to the most probable models (see Section 3.1) on the inner part of the $(u, v)$ plane at $\lambda=1.3 \mathrm{~mm}$. Units are in Jy. Potential $(u, v)$ tracks due to Earth rotation are superposed, with letters indicating antennas. A: APEX/ASTE/ALMA, B: PdB, C: CARMA, H: Hawaii (incl. JCMT), L: LMT, S: SMTO, V: PV.

where $I(x, y)$ is the model intensity distribution with $x$ and $y$ aligned east and north, respectively. We characterize the variation in model visibilities via the standard deviation $\sigma(u, v)$ amongst all models in the ensemble. While we initially ignore the effects of interstellar scattering, it can be included by multiplying the visibilities by a unit-normalized elliptical Gaussian centered at the origin (half width at half maximum of $7.0 \times 3.8 \mathrm{G} \lambda$, position angle $170^{\circ}$ east of north, based on Bower et al. 2006).

Figure 1 shows the scatter in predicted model visibilities, $\sigma(u, v)$. The peak standard deviation occurs at a baseline length of approximately $3 \mathrm{G} \lambda$ at $\lambda=1.3 \mathrm{~mm}(\sim 4000 \mathrm{~km})$ aligned with the accretion disk major axis. Since the disk orientation $(\xi)$ is not known a priori, the optimal baseline orientation is unknown.

Not all models are equally likely, however. The Doeleman et al. (2008b) detections on the JCMT-SMTO and SMTOCARMA baselines as well as total flux density measurement with the CARMA interferometer place constraints on RIAF models. Following the approach of Broderick et al. (2008), we compute the probability $p(a, \theta, \xi)$ for every model based on the Doeleman et al. (2008b) detections, including a $\sin \theta$ prior on the inclination. Restricting the ensemble of models to those with $p \geqslant 0.01 p_{\max }$ (or approximately one-quarter of the total set of models), we can obtain the scatter in the predicted correlated flux densities of the most probable models, $\sigma_{p}(u, v)$ (Figure 2). We find that the largest scatter in models still occurs at baseline lengths near $3 \mathrm{G} \lambda$, but at orientations substantially different from that of the JCMT-SMTO detection (labeled "HS" in Figure 2), which has already placed a stringent constraint on models in its region of $(u, v)$ space.

\subsection{Implications for Specific Baselines}

Figure 3 shows predicted correlated flux densities on potential millimeter VLBI baselines for the probable RIAF models. Each pair of telescopes produces visibility measurements along a range of projected baseline lengths due to the Earth's rotation. These correlated flux densities have been corrected for interstellar scattering.

Detections have already been obtained on the HawaiiSMTO and SMTO-CARMA baselines, although Sgr A* was 




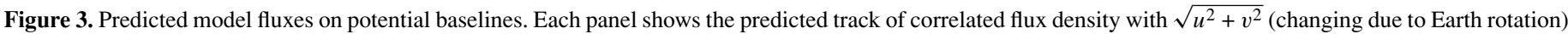



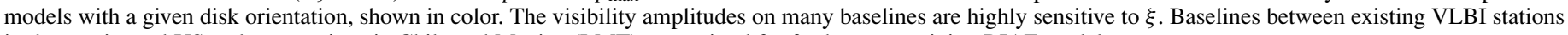
in the continental US and new stations in Chile and Mexico (LMT) are optimal for further constraining RIAF model parameter space.

not detected on the Hawaii-CARMA baseline (Doeleman et al. 2008b). It is likely that the next millimeter VLBI observations of Sgr A* will occur on an array containing these same three stations, although with the inclusion of a phasedarray processor at Hawaii to sum the signals from the JCMT, CSO, and SMA. The increased sensitivity should allow Sgr A* to be detected on the Hawaii-SMTO baseline with a higher signal-to-noise ratio, tightening the constraints on RIAF model parameters especially if Sgr A* can be detected on scans when it is low to the horizon from Arizona, where the range of predicted model flux densities is larger. The Hawaii-CARMA baseline will be more sensitive than the Hawaii-SMTO baseline once the planned array phasing capability is added to CARMA, although the range in predicted model flux densities is somewhat smaller on Hawaii-CARMA. Because the predicted flux densities on the Hawaii-CARMA and Hawaii-SMTO baselines are similar due to their location in approximately the same location of the $(u, v)$ plane, strongly constraining key black hole and accretion disk parameters will necessitate obtaining flux density measurements on other baselines as well. However, measurements on the Hawaii-CARMA baseline may be important to establish the validity of RIAF models of Sgr A* in general and rule out simpler, less physically motivated models, such as a Gaussian or a ring.

The optimal baselines for further constraining model parameter space are SMTO-Chile, CARMA-Chile, and LMT-Chile, which are in the preferred zone of baseline lengths and at a position angle nearly orthogonal to that of HawaiiSMTO. Many of the models predict flux densities of well over $500 \mathrm{mJy}$ (some even above $1 \mathrm{Jy}$ ) on the SMTO-Chile and CARMA-Chile baselines. These flux densities should be detectable even if the Chilean station consists of a single 10 or $12 \mathrm{~m}$ telescope, such as APEX, ASTE, or a single ALMA dish.

Of the three parameters which we consider in this work, millimeter VLBI will most easily be able to constrain the orientation of an RIAF disk. Disk emission comes predominantly from the Doppler-brightened approaching side and is elongated perpendicular to the projected disk major axis when inclined relative to the line of sight. Because the locations of existing and potential millimeter telescopes define preferred directions on the sky for measuring this emission, baseline correlated flux densities depend strongly on disk orientation. The scatter within the predicted correlated flux density for each baseline is much smaller when restricted to one value of $\xi$ than when the entire model parameter space is considered as a whole (Figure 3 ).

Constraining the disk orientation of $\mathrm{Sgr} \mathrm{A}^{*}$ may be a necessary first step toward extracting the spin and inclination of the system. For a given orientation, increasing the black hole spin or the inclination of the system causes the emitting region to appear smaller, raising the correlated flux density on longer baselines (Figure 4). Indeed, if the disk orientation can be determined, the existing Doeleman et al. (2008b) data may already place strong constraints on the spin and inclination Broderick et al. (2008).

\subsection{Potential Complications}

The flux density of $2.4 \mathrm{Jy}$ at $230 \mathrm{GHz}$ detected by Doeleman et al. (2008b) is low compared to measurements with the SMA (Marrone et al. 2007, 2008) and high compared to measurements with the Berkeley-Illinois-Maryland Association (BIMA) array (Zhao et al. 2003; Bower et al. 2005) and a PV-PdB VLBI measurement at $215 \mathrm{GHz}$ (Krichbaum et al. 1998). Sgr A* exhibits variability, although the consistent fluxes measured on two consecutive days by Doeleman et al. (2008b) suggest that Sgr A* was observed in its quiescent state. Since the variability mechanism is not understood, it remains unclear how variability in the total flux density of Sgr A* at millimeter wavelengths will affect the correlated flux densities measured on diverse VLBI baselines. Multiple epochs of observation will be essential to exploring the link between total flux variability and spatial structure around Sgr A*. It will also be important to obtain contemporaneous measurements of fluxes on a variety of baselines as well as a simultaneous "zero-spacing" flux density, preferably from a connected-element interferometer.

Variable and elevation-dependent antenna gains may limit the accuracy to which telescope flux scales can be derived, introducing systematic errors into calculations of correlated flux densities. These systematic errors may be difficult to characterize accurately and may limit the ability of baseline-based quantities to distinguish between different models. Closure amplitudes are robust against station-based gain variations and will provide model constraints with significantly reduced systematic errors. If observations are taken with at least four telescopes, closure amplitudes can be constructed from ratios of baseline visibilities $\left(A_{a b c d}=\left|V_{a b}\right|\left|V_{c d}\right|\left|V_{a c}\right|^{-1}\left|V_{b d}\right|^{-1}\right.$, where subscripted letters identify telescopes). 


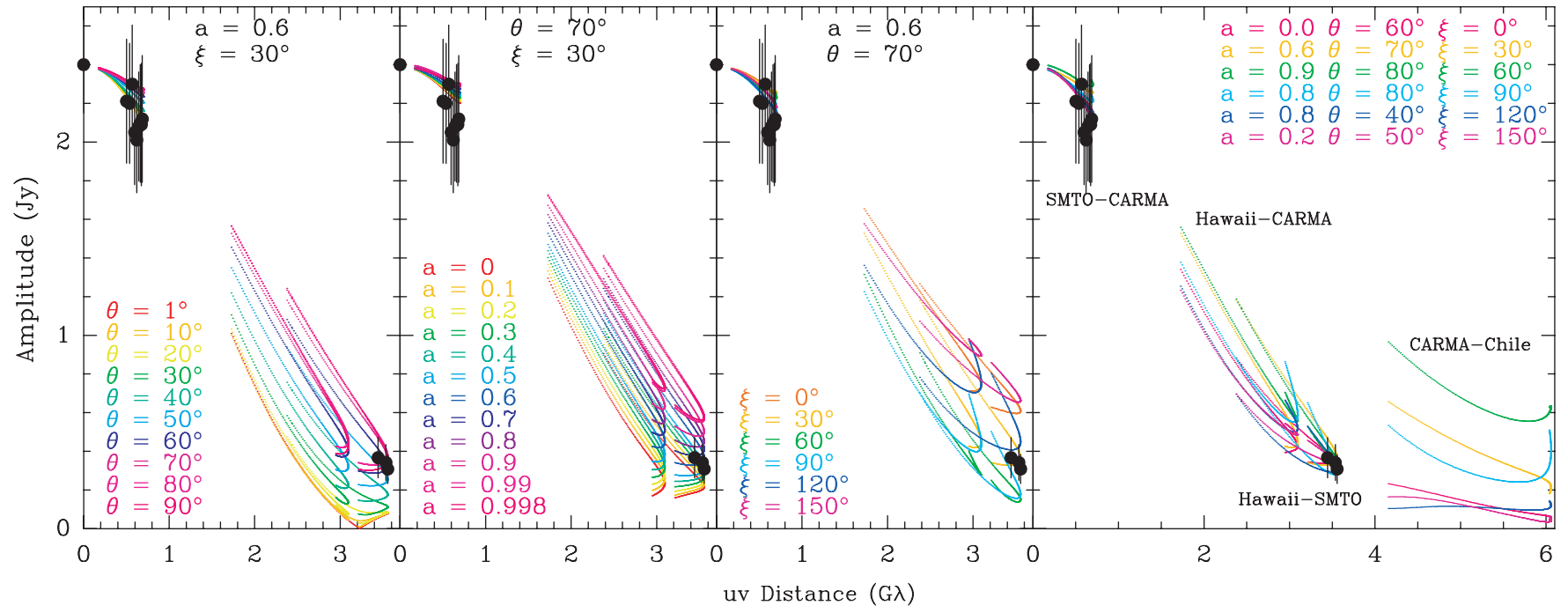

Figure 4. Left three panels: correlated baseline flux density plots on baselines between Hawaii, SMTO, and CARMA for a likely model $\left(a=0.6, \theta=70^{\circ}, \xi=30^{\circ}\right)$, varying one parameter at a time. Data from the second day of observations by Doeleman et al. (2008b) are superposed. Increasing the black hole spin and inclination predicts greater flux densities on longer baselines. Rotation of the disk emission in the plane of the sky can cause the predicted flux density to increase or decrease depending on the baseline orientation. Right panel: the same for six different likely models showing the widely divergent predicted flux densities on the CARMA-Chile baseline.



Figure 5. Predicted model closure amplitudes for all probable RIAF models over the time range for which Sgr A* is above $5^{\circ}$ elevation from at least four of the five Western hemisphere stations. Colors are as in Figure 3. Small jumps in the Hawaii-SMTO-Chile-CARMA panel are artifacts in precision when the Hawaii-Chile baseline passes through a null. A single closure amplitude can also be formed by using two telescopes at the same site, such as CARMA. Closure amplitudes are highly sensitive to $\xi$ and are robust against flux calibration errors.

The models predict a wide range of closure amplitudes on quadrangles of four telescopes, as shown in Figure 5. While even a single measurement of a closure amplitude will be useful, multiple measurements of closure amplitudes on a quadrangle with time will have even greater power in further constraining model parameter space, since different models can predict strongly differing closure amplitudes over a night of observations. As is the case for baseline visibilities, closure amplitudes are most highly sensitive to variations in $\xi$ among the most probable models. From a closure amplitude perspective, either a Chilean telescope or the LMT would make an excellent fourth station in an observing array, although the low predicted correlated flux densities on the Hawaii-Chile baseline may make measurement of a second closure amplitude on an array consisting only of Hawaii, SMTO, CARMA, and Chile difficult without the use of phased-ALMA as the Chilean station.
An alternative observational strategy in the absence of a fourth telescope site would be to use two different telescopes at the same location, for instance at the CARMA site or in Hawaii. A single nontrivial closure amplitude can be formed from the resulting visibilities. The closure amplitude resulting from two CARMA antennas, the SMTO, and Hawaii has less power to differentiate among models than other four-antenna arrays (Figure 5), but multiple detections over a single scan may still be useful. The redundant information provided by the double baselines between the CARMA site and another antenna will also aid in the detection of fringes and reduce the systematic uncertainty in correlated flux densities on the CARMA baselines. Given the nondetection of Sgr A* on the JCMT-CARMA baseline by Doeleman et al. (2008b), it is likely that this strategy would require the use of a phased-array processor at Hawaii.

\section{CONCLUSIONS}

The planned evolution of millimeter VLBI capability over the next few years will place strong constraints on RIAF models of Sgr A* emission. It is important to devise an observing strategy to test the RIAF hypothesis and extract information on the parameters of the putative accretion disk system of Sgr A*, including the disk orientation, inclination, and black hole spin. By identifying differences between models that are consistent with present VLBI observations, we identify four key points to guide future millimeter VLBI observations:

1. There is a preferred zone of baseline lengths near $3 \mathrm{G} \lambda$ where there is enough angular resolution to distinguish between many of the models but not so much resolution that a large amount of flux is lost. The JCMT-SMTO detections are in this zone. The construction of a phasedarray processor for the Hawaiian millimeter telescopes will allow for even tighter constraints to be placed on RIAF model parameters on the Hawaii-SMTO baseline as well as increase the chance of detecting Sgr A* on other baselines to Hawaii.

2. There is a strong correlation between fluxes on the HawaiiSMTO and Hawaii-CARMA baselines, limiting the ability 
of the latter baseline to place extra constraints on RIAF parameters unless a phased-array processor is installed at CARMA, making Hawaii-CARMA a more sensitive baseline than Hawaii-SMTO. Nevertheless, the HawaiiCARMA measurement will be important both for ruling out simpler models (e.g., a Gaussian or annulus) of the emission from Sgr A* that the present VLBI measurements cannot and for testing the RIAF hypothesis.

3. VLBI arrays that include either the LMT or a Chilean telescope provide the best constraints on RIAF models. Predicted flux densities on the SMTO-LMT and CARMALMT baselines are well in excess of $1 \mathrm{Jy}$. Thus, the LMT can significantly enhance Sgr A* VLBI even with a surface not adjusted for maximum accuracy. Likewise, since many models predict flux densities on the SMTO-Chile and CARMA-Chile baselines well in excess of $0.5 \mathrm{Jy}$, inclusion of even a single $10 \mathrm{~m}$ or $12 \mathrm{~m}$ Chilean telescope will result in important model constraints on Sgr A*.

4. Closure amplitudes will be critical, as amplitude calibration errors impose systematic errors on measured baseline flux densities that reduce their ability to constrain RIAF model parameters. At least four telescopes should be used in an observing array in order to obtain closure amplitudes. If no fourth telescope site is available at the time of reobservation, a second antenna at the CARMA site should be included in a $\lambda=1.3 \mathrm{~mm}$ observing array.

We note the important caveat that our analysis assumes that an RIAF accurately and completely describes the emission from Sgr A* at $230 \mathrm{GHz}$. This assumption may be incorrect and is likely at best an approximation to the actual, complex emitting region in $\mathrm{Sgr} \mathrm{A}^{*}$. For these reasons, it will be important to eventually obtain data on as many different millimeterwavelength VLBI baselines as is feasible.

The high-frequency VLBI program at Haystack Observatory is funded through a grant from the National Science Foundation.

\section{REFERENCES}

Bower, G. C., Falcke, H., Wright, M. C. H., \& Backer, D. C. 2005, ApJ, 618, L29

Bower, G. C., Goss, W. M., Falcke, H., Backer, D. C., \& Lithwick, Y. 2006, ApJ, 648, L127

Broderick, A. E., Fish, V. L., Doeleman, S. S., \& Loeb, A. 2008, ApJ, submitted (arXiv:0809.4490)

Doeleman, S. S., Fish, V. L., Broderick, A. E., Loeb, A., \& Rogers, A. E. E. 2008a, ApJ, in press (arXiv:0809.3424)

Doeleman, S. S., et al. 2008b, Nature, 455, 78

Falcke, H., \& Markoff, S. 2000, A\&A, 362, 113

Ghez, A. M., et al. 2008, ApJ, 689, 1044

Igumenschev, I. V., Narayan, R., \& Abramowicz, M. A. 2003, ApJ, 592, 1042

Krichbaum, T. P., et al. 1998, A\&A, 335, L106

Marrone, D. P., Moran, J. M., Zhao, J.-H., \& Rao, R. 2007, ApJ, 654, L57

Marrone, D. P., et al. 2008, ApJ, 682, 373

Schödel, R., Ott, T., Genzel, R., Eckart, A., Mouawad, N., \& Alexander, T. 2003, ApJ, 596, 1015

Yuan, F., Quataert, E., \& Narayan, R. 2003, ApJ, 598, 301

Zhao, J.-H., Young, K. H., Herrnstein, R. M., Ho, P. T. P., Tsutsumi, T., Lo, K. Y., Goss, W. M., \& Bower, G. C. 2003, ApJ, 586, L29 